\title{
Tác động của nhận thức về doanh nghiệp và trách nhiệm xã hội của doanh nghiệp đến ý định mua của người tiêu dùng - Một nghiên cứu trong ngành nước giải khát
}

\section{The effects of perceptions of corporate and corporate social responsibility on consumers' purchasing intention - A study in the beverage industry}

\author{
Nguyễn Hoàng $\operatorname{Sinh}^{1}$, Nguyễn Thị Thanh Tâm ${ }^{1 *}$ \\ ${ }^{1}$ Trường Đại học Mở Thành phố Hồ Chí Minh, Việt Nam \\ ${ }^{2}$ Công ty Xổ số Kiến thiết Kiên Giang, Việt Nam \\ *Tác giả liên hệ, Email: sinh.nh@ou.edu.vn
}

THÔNG TIN

DOI: $10.46223 /$ HCMCOUJS.

econ.vi.17.1.1499.2022

Ngày nhận: 04/03/2021

Ngày nhận lại: 22/03/2021

Duyệt đăng: 31/03/2021

\section{Tù khóa:}

Carroll; nhận thức của người tiêu dùng; thông tin người tiêu dùng tiếp nhận; ý định mua; trách nhiệm xã hội

\section{TÓM TĂT}

Nghiên cứu được thực hiện nhằm đánh giá nhận thức của người tiêu dùng đối với bốn thành phần Trách nhiệm xã hội của doanh nghiệp (gọi tắt là CSR) theo mô hình của Carroll (1991), bao gồm trách nhiệm kinh tế, pháp lý, đạo đức và từ thiện. Nghiên cứu cũng nhằm kiểm định tác động của hai yếu tố mức độ nhận thức của người tiêu dùng về CSR và mức độ thông tin tiếp nhận về CSR đến mối quan hệ giữa nhận thức về doanh nghiệp trách nhiệm xã hội và ý định mua của người tiêu dùng. Nghiên cứu đã đạt được kết quả như sau: thứ nhất, đánh giá tầm quan trọng của người tiêu dùng đối với các thành phần CSR. Thứ hai, những người có mức độ nhận thức về CSR cao có tác động mạnh hơn những người có mức độ nhận thức thấp trong mối quan hệ giữa nhận thức về doanh nghiệp trách nhiệm xã hội và ý định mua. Cuối cùng, những người thường xuyên nhận thông tin trách nhiệm xã hội từ các doanh nghiệp nước giải khát và hiểu thông điệp mà họ gửi đến có tác động mạnh hơn đến ý định mua hàng của người tiêu dùng tại Thành phố Hồ Chí Minh. Các kết quả này là nguồn tham khảo cho các doanh nghiệp trong việc xây dựng, thực hiện CSR nhằm tác động đến ý định người tiêu dùng.

\section{ABSTRACT}

The study was conducted to evaluate consumer perceptions of the four components of Corporate Social Responsibility (referred to as CSR) according to Carroll's (1991) model, including economic and legal, ethical, and philanthropic responsibilities. The study also examines the impact of two factors, the level of consumer perception of social responsibility and the level of information received about CSR on the relationship between consumer's perception of corporate social responsibility and purchasing intentions. The study has shown 
Keywords:

Carroll; customers' perceptions; socially responsible information; corporate social responsibility; purchasing intention some results: first, the order of four responsibilities from customers' opinions differs from that of Carroll (1991). Second, people with a high level of CSR awareness have a stronger impact than those with low levels of understanding of the effects of corporate social responsibility perception and purchasing intent. Lastly, people who regularly receive socially responsible information what they are received to have a positive impact on purchasing intention. These results are the reference source for businesses in their CSR implementation to positively impact consumers.

\section{Giới thiệu}

Trong xu hướng phát triển nhận thức của người tiêu dùng hiện nay, sự công bằng về giá cũng như sự cải tiến của sản phẩm không còn là những mục tiêu hàng đầu khi người tiêu dùng quyết định mua một sản phẩm. Thay vào đó, người tiêu dùng đang quan tâm đến các hoạt động xã hội của doanh nghiệp, hay cụ thể hơn là các hoạt động trách nhiệm xã hội của doanh nghiệp; đây cũng là định hướng mà các nhà doanh nghiệp hướng đến cho sự phát triển lâu dài và bền vững. Khái niệm "Trách nhiệm xã hội của doanh nghiệp" (Corporate Social Responsibility - viết tắt là CSR) là một lĩnh vực rộng và chưa có khái niệm được chấp nhận rộng rãi vì mỗi nhà nghiên cứu có cách lập luận, xác định các thành phần CSR là khác nhau, do chúng được nhìn từ góc độ khác nhau.

Trách nhiệm xã hội của doanh nghiệp ngày nay là một hiện tượng toàn cầu. Hầu hết người tiêu dùng đều đồng ý rằng trong quá trình chinh phục các mục tiêu kinh doanh, doanh nghiệp cũng nên đồng thời thực hiện các nỗ lực CSR. Tuy nhiên, theo lời của Idowu và Filho (2009) thì có một khoảng cách tồn tại giữa sự hiểu biết của chúng ta về cách các doanh nghiệp trong các môi trường chính trị khác nhau, bối cảnh sinh thái và hoàn cảnh văn hóa trên thế giới khác nhau nên họ có thể hiểu, nhận thức và thực hiện trách nhiệm xã hội khác nhau. Chìa khóa cho vấn đề này là những phản ứng của người tiêu dùng đối với hành động của công ty trong việc thực hiện và công bố thông tin CSR. Trong khi đó, theo nghiên cứu mới nhất của Ha (2019), trong 100 công ty niêm yết ở Việt Nam, có 12 công ty không cung cấp thông tin nào liên quan đến trách nhiệm xã hội và chỉ có 14 công ty công bố thông tin đạt trên $80 \%$ theo yêu cầu. Đều này có thể tại Việt Nam, do chưa có nhiều nghiên cứu về tác động của các thông tin CSR đến nhận thức người tiêu dùng. Vì vậy, vấn đề thực hiện CSR ở Việt Nam vẫn là một đề tài đáng quan tâm.

Nghiên cứu này nhằm: (1) đánh giá mức độ nhận thức của người tiêu dùng đối với bốn loại trách nhiệm trong tháp CSR của Carroll (1991), (2) tìm hiểu mức độ ảnh hưởng của nhận thức người tiêu dùng về CSR và mức độ thông tin tiếp nhận CSR đến mối quan hệ giữa nhận thức về doanh nghiệp trách nhiệm xã hội và ý định mua của họ.

\section{Cơ sở lý thuyết và mô hình nghiên cứu}

\subsection{Trách nhiệm xã hội của doanh nghiệp}

Trước những năm 1950, trách nhiệm xã hội của doanh nghiệp thường được gọi là trách nhiệm xã hội (SR) hơn là trách nhiệm xã hội của doanh nghiệp (CSR) trong nhiều năm liền. Điều này có thể là do chưa thật sự thống nhất chung về khái niệm. Năm 1953, Bowen đã xuất bản cuốn sách "Social Responsibilities of the Businessman" mang tính bước ngoặt đánh dấu sự khởi đầu của 
thời kỳ hiện đại của chủ đề này. Sự phân tích của Bowen với CSR xuất phát từ niềm tin rằng hàng trăm doanh nhân lớn nhất vào thời điểm đó là trung tâm quyền lực và ra quyết định quan trọng, hành động của những người này đã ảnh hưởng đến cuộc sống của nhiều công dân theo nhiều cách.

Frederick (1960) đã khẳng định rằng có ba ý tưởng cốt lõi trong CSR: (1) hoạt động của các nhà quản lý doanh nghiệp với tư cách là bên tác động đến lợi ích của cộng đồng, (2) ý tưởng về việc cân bằng các nguồn lực của công ty và (3) chấp nhận từ thiện như là một biểu hiện của sự hỗ trợ kinh doanh vì những lý do chính đáng. Đến năm 1971, Hiệp hội Cam kết phát triển kinh tế (CED) đã đề cao CSR và đòi hỏi doanh nghiệp phải đảm nhận trách nhiệm rộng lớn hơn cho xã hội và phục vụ nhiều giá trị nhân văn hơn và có nghĩa vụ đối với nhóm thành phần trong xã hội ngoài các cổ đông và vượt ra ngoài phạm vi của pháp luật (Jones, 1980).

Năm 1991, Carroll mô tả trách nhiệm kinh tế như là kỳ vọng mà doanh nghiệp sẽ tối đa hóa lợi nhuận cho các cổ đông và trách nhiệm pháp luật như là yêu cầu đối với các doanh nghiệp phải tuân theo các quy định của pháp luật. Trách nhiệm đạo đức có nghĩa là doanh nghiệp phải làm những gì được mong đợi về mặt đạo đức và đúng đạo lý. Trách nhiệm từ thiện có nghĩa là doanh nghiệp nên có chương trình từ thiện với xã hội. Đồng ý kiến, Lantos (2001) giải thích rằng CSR có thể thành chiến lược khi nó là một phần trong kế hoạch quản lý của công ty để tạo ra lợi nhuận, điều đó có nghĩa là công ty sẽ tham gia vào các hoạt động được xem là trách nhiệm xã hội nếu nó mang lại tài chính cho công ty.

European Commission (2011, p. 3) định nghĩa CSR là "một khái niệm, theo đó các công ty tích hợp các mối quan tâm xã hội và môi trường trong hoạt động kinh doanh của họ và trong sự tương tác của họ với các bên liên quan trên cơ sở tự nguyện.” Cụ thể, CSR là một quá trình mà các công ty tích hợp các vấn đề xã hội, môi trường và đạo đức vào các hoạt động kinh doanh và chiến lược của họ trong sự tương tác chặt chẽ với các bên liên quan, vượt trên những yêu cầu của pháp luật và thỏa ước tập thể.

\subsection{Mô hình kim tụ tháp CSR của Carroll}

Carroll (1991) đã mở rộng các lĩnh vực mà ông tin là rất quan trọng khi triển khai CSR trong một tổ chức và đề xuất bốn khía cạnh của CSR: trách nhiệm kinh tế, trách nhiệm pháp lý, trách nhiệm đạo đức và trách nhiệm từ thiện. Carroll đã sắp xếp các khía cạnh này thành một kim tự tháp với phần đáy là nền tảng và quan trọng nhất và cần được ưu tiên hơn các phần ở trên kim tự tháp.

2.2.1. Trách nhiệm tù̀ thiện là những hoạt động của doanh nghiệp đã vượt qua sự kỳ vọng của xã hội bao gồm các hoạt động công ích có lợi cho xã hội, đảm bảo nâng cao chất lượng cuộc sống của cộng đồng và thúc đẩy sự phát triển lành mạnh của toàn xã hội.

2.2.2. Trách nhiệm pháp lý hay còn được gọi là trách nhiệm tuân thủ pháp luật, một phần của bản cam kết giữa doanh nghiệp và xã hội. Nhà nước có trách nhiệm mã hóa những quy tắc, chuẩn mực đạo đức, xã hội vào các văn bản pháp luật. Nó đánh giá sự có lợi hay có hại cho xã hội đơn giản nhất khi đánh giá một doanh nghiệp. Trách nhiệm pháp lý là sự đảm bảo giữa doanh nghiệp và người tiêu dùng, các doanh nghiệp được kỳ vòng sẽ theo đuổi mục tiêu kinh tế trong khuôn khổ mà pháp luật quy định.

2.2.3. Trách nhiệm đạo đức bao gồm những những chuẩn mực, quy tắc được xã hội thừa nhận được kỳ vọng, mong đợi và không bị cấm bởi các thành viên của xã hội không được đề cập đến trong luật pháp. Nó có sự liên hệ chặt chẽ với trách nhiệm pháp lý vì đều chịu ảnh hưởng bởi thói quen, văn hoá của các cá nhân trong xã hội. 
2.2.4. Trách nhiệm kinh tế là trách nhiệm cơ bản nhất, mục đích cơ bản của doanh nghiệp là sản xuất những hàng hóa và dịch vụ mà người tiêu dùng cần và muốn, và tạo ra một mức lợi nhuận chấp nhận được thông qua quá trình kinh doanh. Tất cả những loại trách nhiệm khác của doanh nghiệp đều được xây dựng dựa vào trách nhiệm kinh tế, bởi vì không có nó thì những loại trách nhiệm khác không thể thực hiện được.

\subsection{Nhận thức về CSR của ngưòi tiêu dùng}

Theo Nguyen và Le (2014) nhận định nhận thức về CSR là mức độ đánh giá của Người Tiêu Dùng (NTD) đối với những hoạt động thể hiện CSR, liệu họ có cho rằng những việc làm đó thể hiện sự quan tâm của doanh nghiệp đối với môi trường, cộng đồng, và những việc làm đó có đáng giá để thực hiện hay không.

Theo nghiên cứu của Viện Kenan Châu Á (2010) hầu hết người tiêu dùng Việt Nam hiểu về CSR, phần lớn người được khảo sát nói rằng họ sẽ chọn mua sản phẩm của các công ty có hệ thống sản xuất thân thiện với môi trường, trong khi họ thường từ chối mua sản phẩm của công ty có danh tiếng xấu. Họ không thấy thỏa mãn với vai trò hiện tại của các doanh nghiệp Việt Nam trong việc làm cho xã hội trở nên tốt đẹp hơn và kỳ vọng nhiều hơn vào các doanh nghiệp địa phương. Nghiên cứu của Duong (2015) chỉ ra rằng, mặc dù NTD có nhận thức khá tốt về các vấn đề có liên quan đến CSR nhưng họ chưa quan tâm nhiều đến danh tiếng và việc thực hiện CSR của doanh nghiệp. Hơn nữa, họ vẫn chú ý tới giá khi quyết định mua sản phẩm dịch vụ hơn là việc doanh nghiệp đó có thực hiện tốt CSR hay không.

\subsection{Thông tin tiếp nhận về CSR}

Haron, Yahya, Chambers, Manasseh, và Ismail (2004) chỉ ra rằng, thông tin trách nhiệm xã hội mà NTD tiếp nhận được xác nhận là tích cực đối với hình ảnh công ty và hài hoà với môi trường, xã hội Aerts, Ledoux, Magnan, và Cormier (2008) phân tích các yếu tố quyết định để giải thích sự khác biệt trong việc công bố thông tin trách nhiệm nhiệm xã hội về môi trường trên một số quốc gia. Nghiên cứu cho thấy rằng, ở Hoa Kỳ các doanh nghiệp có mức độ công bố thông tin cao nhất, tiếp theo là Canada, Hà Lan, Pháp, Bỉ và Đức. Kết quả là, NTD có đánh giá các công ty ở Bắc Mỹ tốt hơn so với các công ty tại châu Âu. Điều này cho thấy, việc công bố thông tin có tác động tích cực đến nhận thức NTD và thông tin mà NTD nhận được là có hiệu quả. Theo Gray, Kouhy, và Lavers (1995, p. 50-51) "công bố thông tin trách nhiệm xã hội là quá trình truyền đạt những hoạt động kinh doanh của tổ chức có ảnh hưởng đến môi trường và xã hội tới các cá thể trong xã hội nói riêng và tổng thể xã hội nói chung" như: Báo cáo thường niên, quảng cáo, bài báo cáo hoạt động CSR, báo cáo phát triển cộng đồng, báo cáo môi trường, thông cáo báo chí, băng video và các trang web.

\section{5. Ý định mua của người tiêu dùng}

Ý định mua hàng thể hiện khả năng mà NTD sẽ lập kế hoạch hoặc sẵn sàng mua một sản phẩm hoặc dịch vụ nhất định trong tương lai. Ý định mua của NTD có thể xem như là sự phản ánh của hành vi mua thật sự và ý định mua càng lớn thì mong muốn mua một sản phẩm của NTD càng lớn (Dodds, Kent, \& Dhruv, 1991; Kotler, 2001; Schiffman \& Kanuk, 2007). Khi NTD có ý định mua tích cực, điều này tạo thành một cam kết thương hiệu tích cực và thúc đẩy NTD tiến đến hành động mua thực tế (Fishbein \& Ajzen, 1975). Vì vậy, khi một người quan tâm đến sản phẩm hoặc nhãn hiệu nào đó, họ sẽ có xu hướng tìm hiểu thông tin liên quan đến sản phẩm hoặc nhãn hiệu đó. Ngược lại, nếu một người không quan tâm, họ sẽ có xu hướng bỏ qua bất kỳ thông tin nào.

\subsection{Tác động của Nhận thức về doanh nghiệp trách nhiệm xã hội đến Ý định mua}

Nhận thức của NTD đối với doanh nghiệp thực hiện CSR là đánh giá của NTD đối với những hoạt động thể hiện trách nhiệm xã hội của doanh nghiệp, liệu họ có cho rằng những việc 
làm đó thể hiện sự quan tâm của doanh nghiệp đối với môi trường, cộng đồng và xã hội. Nhiều nghiên cứu đã chỉ ra rằng Nhận thức về doanh nghiệp trách nhiệm xã hội có tác động tích cực đến ý định mua của NTD (Nguyen \& Le, 2014; Yoon \& Lam, 2013). Việc thực hiện tốt CSR không chỉ tác động tích cực đến thành tích về tài chính (Lin, Yang, \& Liou, 2009), giúp gia tăng danh tiếng (Cheung, Tan, Ahn, \& Zhang, 2010), mà nó còn giúp doanh nghiệp có khả năng thu hút được nhiều ứng viên đến ứng tuyển hơn (Wilks, 2002), cũng như giúp duy trì lòng trung thành và thái độ làm việc tích cực của nhân viên đối với tổ chức (Turker, 2009).

\subsection{Tác động của Mức độ thông tin tiếp nhận CSR đến mối quan hệ giữa Nhận thức về doanh nghiệp trách nhiệm xã hội và Ý định mua}

Theo Mohr, Webb, và Harris (2001) ngoài yếu tố giá cả, chất lượng và sự thuận tiện, thì các thông tin trách nhiệm xã hội cũng có tác động đến nhận thức của NTD; tuy nhiên, do mức độ khó khăn trong việc công bố thông tin CSR mà NTD vẫn còn nhiều trở ngại khi tiếp nhận thông tin. Nhiều nghiên cứu đã chứng minh việc công bố thông tin trách nhiệm xã hội có tác động đến lợi ích tài chính của doanh nghiệp. Lợi ích tài chính có thể đạt được từ hai nguồn: Tiết kiệm trực tiếp và cải thiện danh tiếng trên thương trường.

Trong các nghiên cứu về công bố thông tin CSR, các tác giả còn quan tâm đến chất lượng thông tin được công bố thông qua đánh giá chất lượng từng nội dung được công bố và chất lượng hình thức công bố. Có nhiều tác giả đã kết hợp việc đánh giá chất lượng hình thức công bố thông tin để đánh giá công bố thông tin CSR như các nghiên cứu của Toms (2000), Clarkson, Li, Gordon, và Florin (2008). Đánh giá kết hợp giữa đo lường số lượng, chất lượng nội dung và chất lượng thông tin công bố được xem là toàn diện hơn những phương pháp đo lường chỉ dựa trên số lượng thông tin.

\subsection{Tác động của Mức độ nhận thức về CSR đến mối quan hệ giữa Nhận thức về doanh nghiệp trách nhiệm xã hội và Ý̉ định mua}

Viện Kenan Châu Á (2010) đã đưa ra một hướng mới khi đặt ra câu hỏi về trách nhiệm cá nhân (Yoon \& Lam, 2013) khi kết quả khảo sát của nghiên cứu cho thấy mức độ hiểu biết của NTD về CSR có tác động đến nhận thức và thái độ đối với doanh nghiệp trách nhiệm xã hội và sản phẩm của doanh nghiệp và hành vi NTD. Trong khi có rất nhiều nghiên cứu về nhận thức của NTD về CSR tác động đến ý định mua (Huang, Cheng, \& Chun, 2017; Nguyen \& Le, 2014) nhưng chưa đề cập đến việc Mức độ nhận thức của NTD về CSR có ảnh hưởng đến mối quan hệ giữa Nhận thức NTD đối với các doanh nghiệp trong ngành nước giải khát trách nhiệm xã hội và Ý định mua của NTD hay không và những người có mức độ nhận thức về CSR cao (hoặc thấp) sẽ có tác động như thế nào đối với mối quan hệ này.

Với mục tiêu tìm hiểu mối quan hệ giữa nhận thức về CSR của khách hàng và ý định mua, năm 2010, một nhóm nghiên cứu tại Viện Kenan Châu Á (2010) đã thực hiện một nghiên cứu về "Hành vi và thái độ của NTD đối với CSR" tại Việt Nam từ độ tuổi 20 - 60 sống tại Thành phố Hồ Chí Minh. Kết quả cho thấy, NTD Việt Nam hiểu các khái niệm cơ bản về CSR, xem trọng trách nhiệm pháp lý hơn so với các trách nhiệm khác. Mặt khác, thông tin CSR mà người dân tiếp cận được vẫn còn mơ hồ và việc quảng cáo sản phẩm thông qua CSR vẫn chưa thực sự hiệu quả, nhưng nghiên cứu đã chỉ ra rằng mức độ nhận thức của mỗi cá nhân về CSR khác nhau thì sẽ có nhận thức về các hoạt động CSR mà các doanh nghiệp thực hiện cũng khác nhau dẫn đến hành vi tiêu dùng khác nhau. Mặc dù nghiên cứu chưa đi sâu vào vấn đề này nhưng cũng đã mở ra hướng nghiên cứu mới khi đánh giá yếu tố trách nhiệm cá nhân luôn có tác động đến xu hướng phát triển chiến lược kinh doanh mà các doanh nghiệp mong chờ hiệu quả từ CSR. 


\subsection{Mô hình nghiên cúu}

Xuất phát từ cơ sở lý thuyết, mô hình của nghiên cứu này được phát triển dựa trên mô hình của Viện Kenan Châu Á (2010). Ngoài ra, nghiên cứu này kiểm định tác động của yếu tố mức độ thông tin tiếp nhận về CSR đến mối quan hệ giữa nhận thức về doanh nghiệp và ý định mua của NTD.

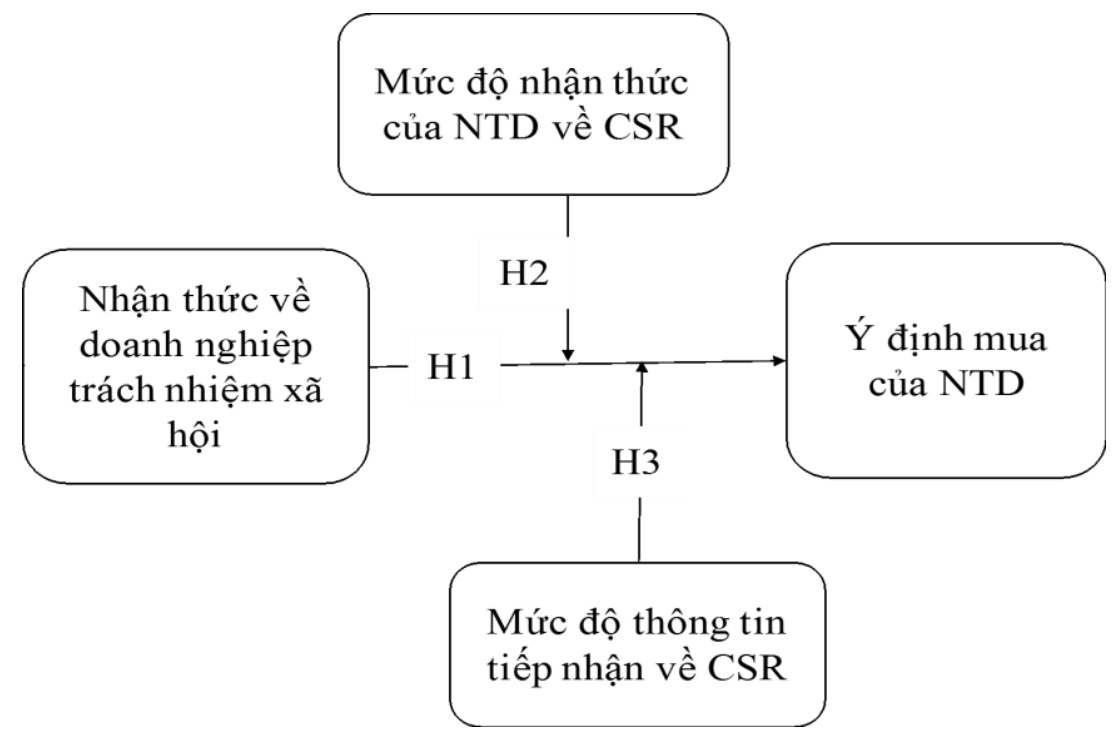

Hình 1. Mô hình nghiên cứu đề xuất

Trong mô hình nghiên cứu này, các yếu tố tác động lên ý định mua của người tiêu dùng bao gồm: Nhận thức về doanh nghiệp trách nhiệm xã hội, Mức độ nhận thức về CSR và Mức độ thông tin tiếp nhận. Trong đó, Mức độ nhận thức về CSR và Mức độ thông tin tiếp nhận không tác động trực tiếp đến ý định mua, mà nó điều tiết sự tác động của yếu tố Nhận thức về doanh nghiệp đối với Ý định mua.

\subsection{Giả thuyết nghiên cứu}

Từ mô hình nghiên cứu (Hình 1 ), giả thuyết được nêu như sau:

H1: Nhận thức về doanh nghiệp trách nhiệm xã hội có tác động tích cực đến Ý định mua hàng của NTD

H2: Mức độ nhận thức của NTD về CSR có ảnh hưởng tích cực đến mối quan hệ giũa Nhận thức về doanh nghiệp trách nhiệm xã hội và Ý định mua của NTD

H3: Mức độ thông tin tiếp nhận về CSR có ảnh hưởng tích cực đến mối quan hệ giữa Nhận thức về doanh nghiệp trách nhiệm xã hội và Ý định mua của NTD

\section{Phương pháp nghiên cứu}

\subsection{Thang do}

Bảng câu hỏi gồm hai phần chính được xây dựng với các thang đo được kết hợp và hiệu chỉnh từ các nghiên cứu trước: Carroll (1991), Mohr và Webb (2005), Nguyen và Le (2014), Hoang (2016) và Viện Kenan Châu Á (2010).

Phần thứ nhất bao gồm các câu hỏi đo lường Nhận thức của người tiêu dùng về CSR.

Phần thứ hai bao gồm các câu hỏi đo lường các yếu tố: Nhận thức về doanh nghiệp, Mức độ thông tin tiếp nhận về CSR. 


\subsection{Thu thập và xử lý dũ liệu}

376 phản hồi hợp lệ được thu thập từ những NTD nước giải khát tại địa bàn nghiên cứu là Thành phố Hồ Chí Minh. Nhằm đạt được mục tiêu nghiên cứu, các phương pháp phân tích dữ liệu sử dụng trong nghiên cứu này bao gồm: thống kê mô tả dữ liệu, đánh giá độ tin cậy của thang đo, phân tích nhân tố khám phá, phân tích nhân tố khẳng định, phân tích mô hình cấu trúc (SEM), phân tích cấu trúc đa nhóm và tác động của biến điều tiết.

\section{Kết quả nghiên cứu}

\subsection{Thống kê mô tả mẫu}

Theo kết quả phân tích dữ liệu bằng SPSS thì trong 376 người tham gia khảo sát: có 161 người là nam (chiếm 42.8\%) và 215 người là nữ (chiếm $51.2 \%$ ). Cho thấy tỷ lệ giới tính của NTD trong mẫu nghiên cứu là tương đối đồng đều.

Tỷ lệ độ tuổi trong mẫu phân bố: nhóm tuổi có tỷ lệ cao nhất là từ 23 - 35 (54.5\%), tiếp đến là $35-50(31.4 \%), 16-22(10.1 \%)$ và trên $50(4 \%)$.

Về yếu tố trình độ học vấn: tỷ lệ cao nhất là bậc đại học $(51.9 \%)$, trung cấp/cao đẳng (26.9\%), sau đại học $(16 \%)$ và thấp nhất là cấp 03 trở xuống $(5.3 \%)$.

Về nghề nghiệp: chiếm tỷ lệ cao nhất là nhân viên văn phòng $(35.1 \%)$, công chức nhà nước $(28.5 \%)$, sau đó là học $\sinh / \sinh$ viên $(17.3 \%)$ và thương nhân/doanh nhân $(11.4 \%)$, thấp nhất là lao động kỹ thuật $(7.7 \%)$.

Về thu nhập: cao nhất là thu nhập từ 10 - 15 triệu (38.0\%), 05 - 10 triệu (35.9\%), dưới 05 triệu $(15.4 \%)$, từ $15-20$ triệu $(5.9 \%)$ và thấp nhất là trên 20 triệu $(4.8 \%)$.

Về nguồn tiếp nhận thông tin: cao nhất là Internet (37.5\%), tivi (23.4\%), kế đến là quảng cáo trên tạp chí $(11.2 \%)$, qua các Báo cáo việc thực hiện trách nhiệm xã hội của doanh nghiệp $(9 \%)$ và nguồn Báo chí và các nguồn khác là thấp nhất (đều $8.5 \%)$.

\subsection{Thống kê mô tả tầm quan trọng CSR}

Đánh giá tầm quan trọng của các thành phần trách nhiệm xã hội (kinh tế, pháp lý, đạo đức, từ thiện) từ 01 đến 04 (từ quan trọng nhất đến ít quan trọng nhất): có 112/376 (29.8\%) đánh giá trách nhiệm đạo đức là quan trọng nhất, thứ hai là trách nhiệm pháp lý với 164/376 (43.6\%), thứ ba là trách nhiệm kinh tế với 133/376 (35.4\%) và ít quan trọng nhất là trách nhiệm từ thiện 210/376 (55.9\%).

\section{Bảng 1}

Thứ tự các thành phần trách nhiệm xã hội của doanh nghiệp

\begin{tabular}{|l|c|c|c|c|}
\hline \multicolumn{1}{|c|}{ Tầm quan trọng } & $\mathbf{1}$ & $\mathbf{2}$ & $\mathbf{3}$ & $\mathbf{4}$ \\
\hline Trách nhiệm kinh tế & $22.9 \%$ & $14.9 \%$ & $\mathbf{3 5 . 4 \%}$ & $26.9 \%$ \\
\hline Trách nhiệm pháp lý & $29.7 \%$ & $\mathbf{4 3 . 6 \%}$ & $16.8 \%$ & $9.8 \%$ \\
\hline Trách nhiệm đạo đức & $\mathbf{2 9 . 8 \%}$ & $32.2 \%$ & $30.6 \%$ & $7.4 \%$ \\
\hline Trách nhiệm từ thiện & $17.6 \%$ & $9.3 \%$ & $17.2 \%$ & $\mathbf{5 5 . 9 \%}$ \\
\hline \multicolumn{1}{|c|}{ Tổng } & $100 \%$ & $100 \%$ & $100 \%$ & $100 \%$ \\
\hline
\end{tabular}

Nguồn: Kết quả phân tích dữ liệu 


\subsection{Kiểm định thang đo}

Các biến trong mô hình nghiên cứu gồm các thang đo sau:

- Nhận thức về các doanh nghiệp trách nhiệm xã hội, gồm 12 biến, bao gồm: 03 biến đo nhận thức của người tiêu dùng về các hoạt động kinh tế của doanh nghiệp được đánh số lần lượt từ HDKT1 đến HDKT3, 03 biến hoạt động pháp luật từ HDPL1 đến HDPL3, 03 biến hoạt động đạo đức từ HDDD1 đến HDDD3, 03 biến hoạt động từ thiện từ HDTT1đến HDTT3;

- Ý định mua của NTD gồm 03 biến được đánh số từ YD1 đến YD3;

- Mức độ nhận thức của NTD về CSR gồm 15 biến, bao gồm: 04 biến nhận thức về hoạt động kinh tế từ KT1 đến KT3, 04 biến hoạt động pháp luật từ PL1 đến PL3, 03 biến hoạt động đạo đức từ DD1 đến DD3, 04 biến hoạt động từ thiện từ TThien1 đến TThien3;

- Mức độ thông tin tiếp nhận về CSR gồm 03 biến đánh số lần lượt từ TT1 đến TT3.

Sau khi tiến hành kiểm định, tất cả các thang đo có hệ số Cronbach Alpha từ 0.772 đến 0.932 trên 0.6 , nghĩa là 33 biến quan sát đều phù hợp.

\subsubsection{Phân tích nhân tố khám phá}

Kết quả phân tích nhân tố cho thấy chỉ số KMO là $0.827>0.5$ đã đạt yêu cầu, điều này chứng tỏ dữ liệu dùng để phân tích nhân tố là hoàn toàn thích hợp. Kết quả kiểm định Barlett's là $3,015.452$ với mức ý nghĩa $\mathrm{p}=0.000<0.05$, các biến có tương quan với nhau và thỏa điều kiện phân tích nhân tố.

Thực hiện phân tích nhân tố theo phương pháp trích xuất Principal Axis Factoring và phép quay Promax với kết quả giá trị tổng phương sai trích là $67.372 \%>50 \%$; Giá trị hệ số Eigenvalues của các nhân tố là $1.198>1$ đạt yêu cầu.

Các hệ số tải nhân tố đều lớn hơn 0.5 , và không có trường hợp biến nào cùng lúc tải lên cả hai nhân tố với hệ số tải gần nhau. Nên các nhân tố đảm bảo được giá trị hội tụ và phân biệt khi phân tích EFA.

\subsubsection{Phân tỉch nhân tố khẳng định $C F A$}

Trong nghiên cứu này, theo kết quả sau khi chạy AMOS 20 ta có $\mathrm{CMIN}=108.035$; $\mathrm{df}=$ 80; $\mathrm{CMIN} / \mathrm{df}=1.350<2 ; \mathrm{p}=0.020<5 \%$; RMSEA $=0.031<0.07 ; \mathrm{CFI}=0.991>0.9 ;$ GFI $=$ $0.963>0.9 ; \mathrm{TLI}=0.988>0.9 ;$ kết luận chúng đạt tính đơn hướng và dữ liệu phù hợp.

Kết quả hệ số tin cậy tổng hợp $(\mathrm{CR})$ của nghiên cứu đều lớn hơn 0.7 và hệ số phương sai trích (AVE) đều lớn hơn 0.5 đã đạt yêu cầu (Hair, Black, Babin, \& Anderson, 2010), thang đo của 05 nhân tố rất đáng tin cậy.

Chỉ số MSV đều nhỏ hơn chỉ số AVE và chỉ số SQRTAVE đều lớn hơn hệ số tương quan đủ đều kiện tính phân biệt.

\subsection{Mô hình cấu trúc tuyến tính (SEM)}

Mô hình cấu trúc tuyến tính SEM được thực hiện để kiểm định giả thuyết $\mathrm{H} 1$. Theo kết quả (Hình 4), với độ tin cậy $95 \%$ giả thuyết $\mathrm{H} 1$ được chấp nhận với $\mathrm{p}=0.000<0.05$ có ý nghĩa thống kê. Nghĩa là, Nhận thức về doanh nghiệp trách nhiệm xã hội có tác động dương đến Ý định mua của NTD với giá trị hồi quy đã chuẩn hóa là 0.767 . 


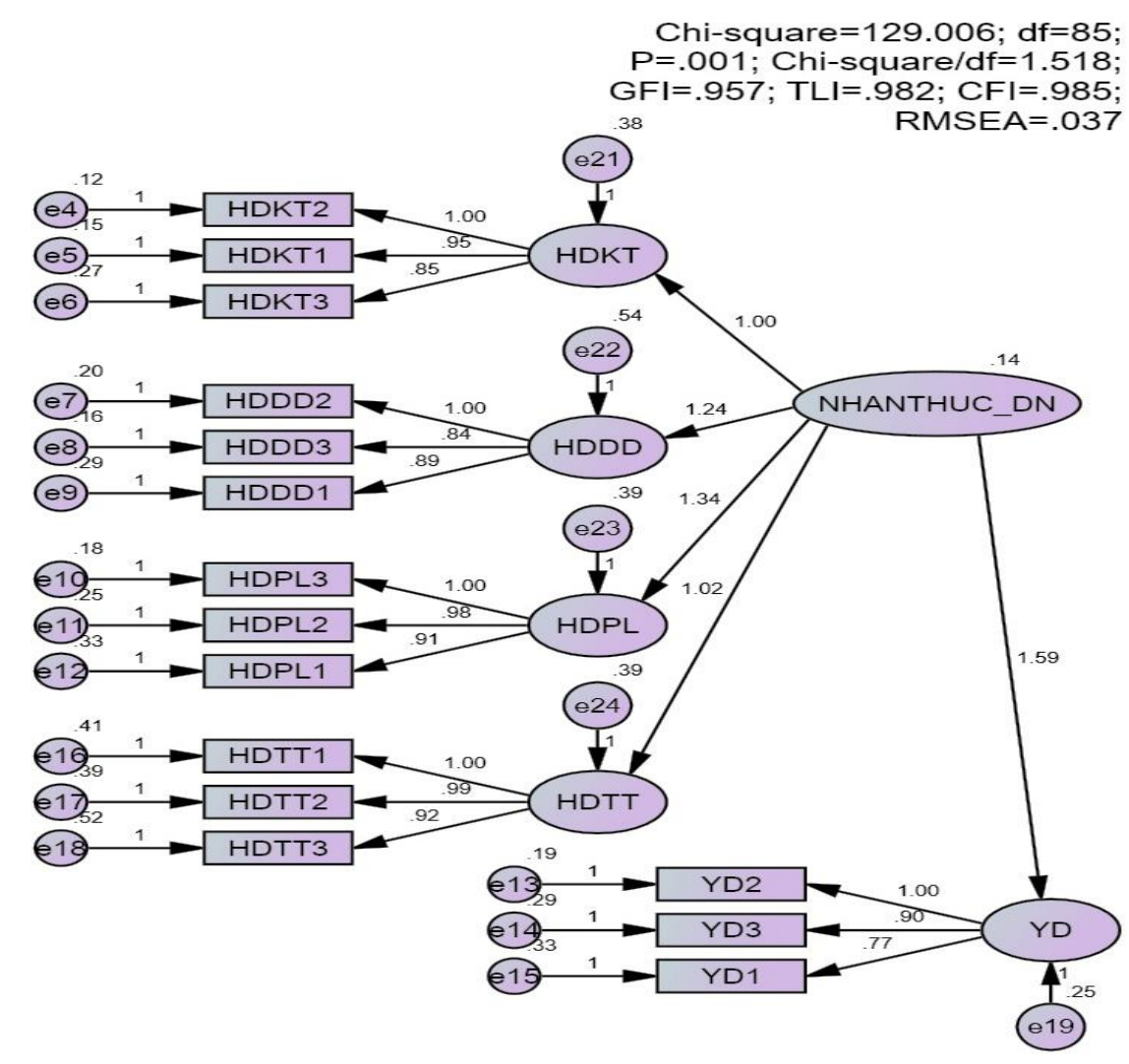

Hình 2. Kết quả SEM

\subsection{Phân tích cấu trúc đa nhóm}

Để đánh giá mối quan hệ giữa hai nhân tố có sự khác biệt bởi một nhóm khác hay không (kiểm định các giả thuyết $\mathrm{H} 2$ và $\mathrm{H} 3$ ), nghiên cứu này sử dụng phân tích cấu trúc đa nhóm bằng mô hình khả biến và mô hình bất biến (từng phần).

\subsubsection{Mức độ nhận thức về CSR}

Kết quả kiểm định Chi-square với $\mathrm{p}=0.005<0.05$ kết luận có sự khác biệt về mối quan hệ giữa Nhận thức về doanh nghiệp trách nhiệm xã hội và Ý định mua của NTD giữa nhóm có mức độ nhận thức về CSR thấp và cao. Cụ thể, với nhóm có mức độ nhận thức cao (hệ số hồi quy đã chuẩn hóa là 0.950 ) mối quan hệ giữa Nhận thức về doanh nghiệp trách nhiệm xã hội và Ý định mua của NTD mạnh hơn nhóm có mức độ nhận thức thấp (hệ số hồi quy đã chuẩn hóa là 0.815 ).

\subsubsection{Mức độ thông tin tiếp nhận}

Với $\mathrm{p}=0.04<0.05$, cho thấy có sự khác biệt trong mối quan hệ giữa Nhận thức về doanh nghiệp trách nhiệm xã hội đến Ý định mua của NTD giữa nhóm có mức độ tiếp nhận thông tin ít và nhiều. Cụ thể, mối quan hệ giữa Nhận thức về doanh nghiệp trách nhiệm xã hội đến Ý định mua của NTD của những người nhận thông tin nhiều (hệ số hồi quy đã chuẩn hóa là 0.805) mạnh hơn những người nhận thông tin ít (hệ số hồi quy đã chuẩn hóa là 0.714 ).

\subsection{Hiệu ứng điều tiết}

Mô hình hiệu ứng biến điều tiết Mức độ nhận thức về CSR được diễn tả thành phuơng trình sau:

$$
\mathrm{YD}=\mathrm{p} 1 * \mathrm{X}_{1}+\mathrm{p} 2 * \mathrm{X}_{2}+\mathrm{p} 3 * \mathrm{X}_{3}
$$


Trong đó:

YD: Ý định mua của NTD;

$\mathrm{X}_{1}$ Nhận thức của NTD về doanh nghiệp trách nhiệm xã hội;

$\mathrm{X}_{2}$ Mức độ nhận thức về CSR;

$\mathrm{X}_{3}$ Nhận thức về doanh nghiệp trách nhiệm xã hội x Mức độ nhận thức về CSR;

p1, p2, p3 là hệ số hiệu ứng đều tiết lần lượt của $X_{1}, X_{2}, X_{3}$. trình sau:

Mô hình hiệu ứng biến điều tiết Mức độ thông tin tiếp nhận được diễn tả thành phuơng

$$
\mathrm{YD}^{\prime}=\mathrm{h} 1 * \mathrm{Z}_{1}+\mathrm{h} 2 * \mathrm{Z}_{2}+\mathrm{h} 3 * \mathrm{Z}_{3}
$$

Trong đó:

YD': Ý định mua của NTD;

$Z_{1}$ Nhận thức của NTD về doanh nghiệp trách nhiệm xã hội;

$Z_{2}$ Mức độ thông tin tiếp nhận;

$Z_{3}$ Nhận thức về doanh nghiệp trách nhiệm xã hội x Mức độ thông tin tiếp nhận;

h1, h2, h3 là hệ số hiệu ứng đều tiết lần lượt của $Z_{1}, Z_{2}, Z_{3}$.

Theo kết quả, có sự tương tác giữa nhóm ngưới có mức độ nhận thức về CSR cao và thấp; cụ thể, những người có ý định mua hàng nhiều hơn khi họ nhận thức về CSR cao hơn và nhận thức về doanh nghiệp trách nhiệm xã hội tốt hơn (biến điều tiết "Nhận thức về doanh nghiệp trách nhiệm xã hội x Mức độ nhận thức về CSR" tác động đến biến phụ thuộc "Ý định mua của người tiêu dùng" có ý nghĩa thống kê, $\mathrm{p}=0.02<0.05)$. Tương tự, những người có ý định mua hàng nhiều hơn khi họ nhận được nhiều thông tin hơn và có nhận thức về doanh nghiệp trách nhiệm xã hội tốt hơn (biến điều tiết "Nhận thức về doanh nghiệp trách nhiệm xã hội x Mức độ thông tin tiếp nhận" tác động đến biến phụ thuộc "Ý định mua của người tiêu dùng" có ý nghĩa thống kê, $\mathrm{p}=0.00<0.05)$. Vậy, giả thuyết $\mathrm{H} 2, \mathrm{H} 3$ được chấp nhận.

\subsection{Thảo luận kết quả nghiên cứu}

Kết quả phân tích bằng phần mềm SPSS 20 , AMOS 20 cho thấy giả thuyết $\mathrm{H} 1 \mathrm{a}$ : Trách nhiệm kinh tế có tác động cùng chiều với Nhận thức về doanh nghiệp trách nhiệm xã hội với hệ số hồi quy đã chuyển hóa là 0.521 . Tương tự, giả thuyết H1b, H1c, H1d: Trách nhiệm pháp lý, Trách nhiệm đạo đức và Trách nhiệm từ thiện đều có tác động cùng chiều với Nhận thức về doanh nghiệp trách nhiệm xã hội với hệ số hồi quy đã chuyển hóa lần lượt là $0.626 ; 0.537 ; 0.522$. Ở độ tin cậy $95 \%$ với $\mathrm{p}<0.05$ cho thấy giả thuyết: Nhận thức về doanh nghiệp trách nhiệm xã hội có tác động dương đến Ý định mua của NTD với giá trị hồi quy đã chuẩn hóa là 0.767 . Nhận thức về doanh nghiệp càng cao thì ý định mua đối với các sản phẩm của các doanh nghiệp này càng cao. Điều này có nghĩa rằng, NTD rất quan tâm đến các hoạt động của các doanh nghiệp nước giải khát đặt biệt là khi các doanh nghiệp lớn như Pepsico Việt Nam và Tập đoàn Tân Hiệp Phát luôn chú trọng tập trung vào hình ảnh thương hiệu để thu hút khách hàng. Nhận thức về doanh nghiệp càng cao thì ý định mua đối với các sản phẩm của các doanh nghiệp này càng cao. Kết quả nghiên cứu của tác giả phù hợp với nghiên cứu của Carvalho, Sem, và Mota (2010); Viện Kenan Châu Á (2010); Yoon và Lam (2013); Chow và Jian (2012).

Trong đó, nhóm có Mức độ nhận thức của NTD về CSR cao có tác động mạnh mẽ hơn đến mối quan hệ giữa Nhận thức về doanh nghiệp trách nhiệm xã hội trong ngành nước giải khát và Ý 
định mua của NTD (hệ số đã chuẩn hóa 0.950) so với nhóm có Mức độ nhận thức của NTD về CSR thấp (hệ số đã chuẩn hóa 0.815). Ngoài ra, Mức độ thông tin tiếp nhận về CSR có tác động đến mối quan hệ Nhận thức về doanh nghiệp trách nhiệm xã hội và Ý định mua. Điều này có nghĩa là, trong điều kiện tiến hành nghiên cứu tại Thành phố Hồ Chí Minh, với sản phẩm cụ thể là nước giải khát, những người có nhận thức về CSR cao và nhận thông tin về CSR nhiều có sự thay đổi về ý định mua mạnh mẽ hơn khi các doanh nghiệp thực hiện các hoạt động CSR so với nhóm còn lại.

\section{Kết luận và hàm ý quản trị}

Trong tổng số 376 phản hồi thu được, các đáp viên đánh giá tầm quan trọng của các loại trách nhiệm xã hội từ 01 đến 04 (từ quan trọng nhất đến ít quan trọng nhất) như sau: trách nhiệm đạo đức là quan trọng nhất, đứng thứ hai là trách nhiệm pháp lý, đứng thứ ba là trách nhiệm kinh tế và cuối cùng, ít quan trọng nhất là trách nhiệm từ thiện. Kết quả này hoàn toàn ngược lại với quan điểm của Carroll (1991) khi ông cho rằng trách nhiệm kinh tế nên được ưu tiên hơn cả, nhưng đồng quan điểm với nghiên cứu của Chow và Jian (2012) khi khẳng định rằng hầu hết người Malaysia liên kết thuật ngữ CSR với trách nhiệm đạo đức. Đây là yếu tố chiến lược mà các doanh nghiệp phải hướng đến khi sử dụng các phương tiện truyền thông khi quảng bá sản phẩm, đặc biệt là các bên liên quan (nhà cung cấp, cộng đồng, môi trường, NTD...).

Kiểm định Chi-square giữa mô hình bất biến và mô hình khả biến, kết quả ý nghĩa giữa hai mô hình $\mathrm{p}<0.05$, có sự tương tác giữa nhóm người có mức độ nhận thức về CSR cao và thấp; cụ thể, NTD có ý định mua hàng nhiều hơn khi họ nhận thức về CSR cao hơn và nhận thức về doanh nghiệp trách nhiệm xã hội tốt hơn. Tương tự, NTD có ý định mua hàng nhiều hơn khi họ nhận được nhiều thông tin CSR hơn và có nhận thức về doanh nghiệp trách nhiệm xã hội tốt hơn. Đây là một khám phá mới mẽ mà các nghiên cứu trước (Carvalho et al., 2010; Viện Kenan Châu Á, 2010; Yoon \& Lam, 2013) chưa nghiên cứu kỹ.

Cụ thể, về thông tin tiếp nhận CSR, trong nghiên cứu này, NTD nhận định rằng, họ thường xuyên nhận được thông tin về các doanh nghiệp nước giải khát thực hiện trách nhiệm xã hội thông qua internet (37.5\%) và tivi (23.4\%). Kết quả này khác so với kết quả mà Viện Kenan Châu Á (2010) đã công bố khi cho rằng NTD nhận ít thông tin về CSR. Điều này có thể là trong thời gian 10 năm từ năm 2010 đến năm thực hiện nghiên cứu này (năm 2020) các doanh nghiệp nghiên cứu (nước giải khát) đã tích cực sử dụng các phương tiện truyền thông cùng với với sự phát triển mạnh mẽ của công nghệ thông tin giúp NTD tiếp nhận nhiều thông tin CSR hơn. Như vậy, kết quả nghiên cứu này phản ánh sự thành công của việc công bố thông tin CSR mà các doanh nghiệp nước giải khát đã thực hiện trong thời gian gần đây.

\section{Tài liệu tham khảo}

Aerts, W., Ledoux, M., Magnan, M., \& Cormier, D. W. (2008). Attributes of social and human capital disclosure and information asymmetry between managers and investors. Canadian Journal of Administrative Sciences, 26(1), 71-88.

Bowen, H. (1953). Social responsibility of the businessman. New York, NY: Harper \& Row.

Carroll, A. B. (1991). The pyramid of corporate social responsibility: Toward the moral management of organizational stakeholders. Business Horizons, 34(4), 39-48. doi:10.1016/0007-6813(91)90005-G

Carvalho, S., Sem, S., \& Mota, M. (2010). Consumer reactions to CSR: A Brazilian perspective. Journal of Business Ethics, 91(1), 291-310. 
Cheung, Y. L., Tan, W., Ahn, H. J., \& Zhang, Z. (2010). Does corporate social responsibility matter in asian emerging markets? Journal of Business Ethics, 92(3), 401-413.

Chow, M. M., \& Jian, A. Y. (2012). A study of the effect of Corporate Social Responsibility (CSR) towards consumer buying behaviour. Economics and Finance, 31(2), 16-33.

Clarkson, P. G., Li, Y., Gordon, D. R., \& Florin, P. V. (2008). Revisiting the relation between environmental performance and environmental disclosure: An empirical analysis. Accounting, Organizations and Society, 33(4), 303-327.

Dodds, W. B., Kent, M., \& Dhruv, G. (1991). Effects of price, brand, and store information on buyers' product evaluations. Journal of Marketing Research, 28(3), 307-319.

Duong, D. C. (2015). Nhận thức của người tiêu dùng về trách nhiệm xã hội của doanh nghiệp [Consumers' perception of corporate social responsibility]. Tạp chí Kinh tế phát triển, 217(2), 24-32.

European Commission. (2011). Communication from the commission to the European Parliament, the Council, the European economic and social committee and the committee of the regions. Retrieved January 10, 2021, from https://www.europarl.europa.eu/meetdocs/2009_2014/documents/com/com_com(2011)068 1_/com_com(2011)0681_en.pdf

Fishbein, M., \& Ajzen, I. (1975). Belief, attitude, intention, and behavior: An introduction to theory and research. Boston, MA: Addison-Wesley.

Frederick, W. C. (1960). The growing concern over business responsibility. California Management Review, 2(4), 54-61.

Gray, R., Kouhy, R., \& Lavers, S. (1995). Corporate social and environmental reporting: A review of the literature and a longitudinal study of UK disclosure. Accounting, Auditing \& Accountability Journal, 8(2), 47-77.

Ha, T. T. (2019). Thực trạng công bố thông tin trách nhiệm xã hội (CSR) của các công ty niêm yết tại Việt Nam [Current situation of Corporate Social Responsibility (CSR) information disclosure of Vietnamese listed companies]. Tạp chí Phát Triển và Hội Nhập, 46(56), 6874.

Hair, J. F. J., Black, W. C., Babin, B. J., \& Anderson, R. E. (2010). Multivariate data analysis (7th ed.). Upper Saddle River, NJ: Prentice Hall.

Haron, H., Yahya, S., Chambers, A., Manasseh, S., \& Ismail, I. (2004). Level of corporate social disclosure in Malaysia. Singapore: Fourth Asia Pacific Interdisciplinary Research in Accounting Conference.

Hoang, T. T. (2016). Nhận thức của người tiêu dùng Việt Nam về trách nhiệm xã hội của công ty Yến Sào quyết định đến hành vi mua hàng [Customers' awareness of corporate social responsibility of Khanh Hoa salanganes nest company has effect on the consumption behavior]. Tạp chí Khoa học - Công nghệ Thủy sản, 2(3), 107-115.

Huang, M., Cheng, Z., \& Chun, C. I. (2017). The importance of CSR in forming customercompany identification and long-term loyalty. Journal of Services Marketing, 31(1), 63-72.

Idowu, S. L., \& Filho, W. L. (2009). The global practices of corporate social responsibility. Heidelberg, Germany: Springer-Verlag. 
Jones, T. M. (1980). Corporate social responsibility revisited, redefined. California Management Review, 22(3), 59-67.

Kotler, P. (2001). Marketing management. Upper Saddle River, NJ: Pearson Education.

Lantos, G. P. (2001). The boundaries of strategic corporate social responsibility. Journal of Consumer Marketing, 18(7), 595-632.

Lin, C. H., Yang, H. L., \& Liou, D. Y. (2009). The impact of corporate social responsibility on financial performance: Evidence from business in Taiwan. Technology in Society, 31(1), 56-63.

Mohr, L. A., \& Webb, D. J. (2005). The effects of corporate social performance price on consumer response. Journal of Consumer Affairs, 39(1), 121-147.

Mohr, L. A., Webb, D. J., \& Harris, K. E. (2001). Do consumers expect companies to be socially responsible? The impact of corporate social responsibility on buying behavior. The Journal of Consumer Affairs, 35(1), 45-72.

Nguyen, N. P. T., \& Le, X. T. T. (2014). Nhận thức của người tiêu dùng về trách nhiệm xã hội của doanh nghiệp và ý định mua - Một nghiên cứu từ ngành hàng điện máy [Consumers' perception of corporate social responsibility and buying intent - A study from the electronics industry]. Tạp chí Khoa học Trương Đại học Mở Thành phố Hồ Chí Minh, 9(2), 60-79.

Schiffman, L., \& Kanuk, L. (2007). Consumer behavior (9th ed.). Upper Saddle River, NJ: Pearson Prentice Hall.

Toms, J. S. (2000). Environmental management, environmental accounting and financial performance. Oxford, UK: CIMA.

Turker, D. (2009). Measuring corporate social responsibility: A scale development study. Journal of Business Ethics, 85(4), 41-42.

Viện Kenan Châu Á. (2010). Hành vi và thái độ của ngườ tiêu dùng đối với trách nhiệm xã hội doanh nghiệ [Consumer behavior and attitude towards corporate social responsibility]. Ho Chi Minh City, Vietnam: Viện Kenan Châu Á.

Wilks, N. (2002). Good behaviour is its own reward. Professional Engineering, 15(8), 36-37.

Yoon, S., \& Lam, T. (2013). The illusion of righteousness: Corporate social responsibility practices of the alcohol industry. BMC Public Health, 13(1), 76-91.

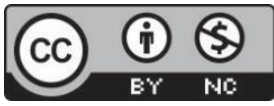

Creative Commons Attribution-NonCommercial 4.0 International License. 